\title{
EFFECTS OF CARBOFURAN ON THE REPRODUCTIVE CAPACITY OF A FRESHWATER SNAIL, RADIX QUADRASI, UNDER LABORATORY CONDITIONS
}

\author{
IMELDAF. PAGULAYAN, GLORIAL. ENRIQUEZ \\ and VIRGINIAS. CARING \\ College of Science, University of the Philippines \\ Diliman, 1001 Quezon City, Philippines
}

\begin{abstract}
The effects of 4 sublethal concentrations of carbofuran $(250,500,1000$ and $2000 \mathrm{ppm})$ on the reproductive capacity of $R$. quadrasi was determined. Results showed that incubation period is delayed and inhibited by 1000 and $2000 \mathrm{ppm}$ carbofuran but not by lower concentrations. The hatching period is longer in treated snails and not all eggs hatch in the 1000 and $2000 \mathrm{ppm}$ treatment. The percentage of hatching is inversely proportional to the carbofuran concentration.

Oviposition was delayed in all the treated stages and at all dosages. The higher the carbofuran concentration, the later the onset of oviposition. The reproductive period is shortened. Fecundity was decreased in snails treated at EMB and SM. However, only the 2000 ppm carbofuran concentration showed an adverse effect on the snails exposure at PSM.
\end{abstract}

\section{INTRODUCTION}

Pesticides are continuously finding widespread use and increasing amounts are being introduced into the aquatic environment. Researchers worry that this increasing use of pesticides and the farming industry's dependence on it may eventually lead to health problems for both consumers of farm products and farm workers (Allmon 1985). Moreover, this will pose a potential hazard not only to human beings but also to aquatic organisms of economic importance (Mane et al. 1979).

Invertebrates constitute a very large portion of the fauna in both the aquatic and terrestrial system. From an ecological viewpoint, these animals play an important role in the energy flow in these ecosystems. The feeding habit of invertebrate animals varies from detritus feeding to herbivorous and carnivorous food consumption. Disruptions caused by pesticide stress in one or more organisms may directly interfere with other inter-dependent components of the food chain (Gunther et al. 1968).

Carbofuran (2,3-dihydro-2,2-dimethyl-7-benzofuranyl-N-methylcarbamate) developed by the Niagara Chemical Division of FMC Corporation is commercially known as Furadan. It is a systemic insecticide, nematicide with broad spectrum activity (Metcalf $e t$ al. 1968; Yu et al. 1974; Cremlyn 1980; Handa 1980) although hydro- 
lyzed rapidly in water. It is an effective contact toxicant and is non-corrosive and compatible with other pesticides (Rangaswany et al. 1976). Carbamate insecticide is a popular choice since it is less toxic, less persistent and has lower residual effects in animals than other classes of pesticides. Nevertheless, it is accumulated to a certain extent.

This work aims to determine the levels of carbofuran concentrations that are toxic or deleterious to the embryonic (EMB), presexually mature (PSM), and sexually mature (SM) R. quadrasi; to determine the effects of sublethal concentrations of carbofuran on the fecundity and reproductive period of the snail; and to determine the particular age group that is most sensitive to carbofuran.

\section{MATERIALS AND METHODS}

\section{Organism}

Radix quadrasi, (Fig. 1) the snail used in this study, were obtained from stock cultures started in 1981 and have been maintained and reared continuously in the Snail Room of the Natural Sciences Research Institute, University of the Philippines, Diliman, Quezon City.

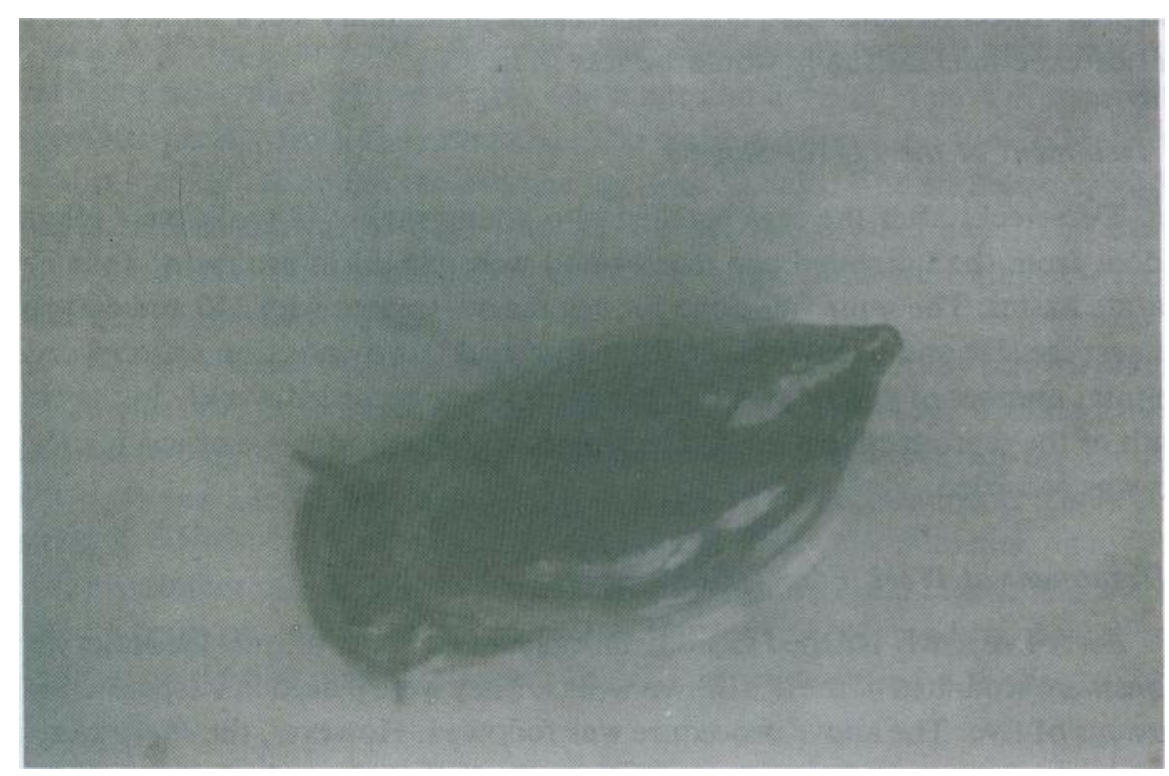

Figure 1. Radix quadrasi 
Laboratory maintenance and culture of snails, maintenance of eggs and young snails followed that of de Lara and Enriquez (1981) and Pagulayan and Darvin (1986).

\section{Preparation of Working Solutions}

Carbofuran in the form of $3 \mathrm{G}$ was prepared by dissolving the granules first in acetone before adding distilled water. The solution was aerated overnight before use. Concentration parts in per million (ppm) were used.

All glasswares and plastic containers were acid washed and rinsed with de-ionized water. Petri dishes were oven-dried before use.

\section{Experimental Set-up}

Thirty egg masses were used as the source of snails that were observed for fecundity and reproductive period. Ten of these egg masses were allowed to hatch under normal conditions (control). The other experimental groups consisting of 10 egg masses each were treated with $250 \mathrm{ppm}$ and $500 \mathrm{ppm}$ carbofuran for a period of one week in separate petri dishes. Two-week treatment durations and 1000 and 2000 ppm concentrations were not used because preliminary work showed that survival of experimental snails would be less than $50 \%$.

\section{A. Treatment at the Larval Stage}

Two weeks after the eggs hatched into young snails, 48 snails were taken at random from the untreated egg masses and were placed in groups of four in 12 separate basins. The same was done for egg masses treated with 250 and $500 \mathrm{ppm}$. The average size and age of the snails at first and last oviposition were recorded. The total number of egg masses laid per group were recorded weekly in the entire length of the reproductive period. The length of the reproductive or egg laying period was recorded.

\section{B. Treatment at Week Four (PSM)}

Forty five newly hatched snails from egg masses under normal laboratory conditions were separated at the age of two weeks. They were placed in 9 separate basins in groups of five. The above procedure was followed. However, the snails were exposed to the four carbofuran concentrations applied at the end of the 4th week to the end of the 6th week after hatching. 
Effects of carbofuran on the reproductive capacity - Imelda F. Pagulayan et al.

\section{Treatment at Week Seven (SM)}

The same procedure was followed except that the snails were treated at the end of the 7 th week to the end of the 9th week after hatching.

In the two-week duration of treatment, the medium was changed after the first week. Possible toxic material in the medium like the feces were removed regularly.

\section{RESULTS}

\section{Effects of Fecundity}

Fecundity is the total number of the eggs produced by the individual during its reproductive period. Reproductive period, on the other hand, is defined as the number of days from the first to the last day of egg-laying.

\section{A. Treatment at Embryonic Stage (EMB)}

Table 1 shows the size range and average at the onset and end of oviposition, the reproductive capacity and the reproductive period of $R$. quadrasi that were hatched from untreated embryos and embryos exposed to 250 and 500 ppm concentrations of carbofuran. The average size length: 10.62, 10.56 and $10.45 \mathrm{~mm}$ for the control, $250 \mathrm{ppm}$ and $500 \mathrm{ppm}$ treatment respectively, are not significantly different from each other. However, the age range and average in days of the treated snails differ greatly from the untreated. The earliest oviposition took place in the control at day 29, although the average age was 39 days. The earliest oviposition for both treatments was 43 days with an average age of 49.5 days. The treated snails oviposited at a much older age but the size of the snails were almost the same as that of the untreated ones.

The average size length at last oviposition differ between the control and the treated snails; for the control it took place at an older age range with an average of 224 days. Oviposition stopped at an average age of 196.5 days for the 250 ppm treatments; and 193 days as average age for the $500 \mathrm{ppm}$ treatment. There is no significant difference in the size and average for the two treatments.

The number of egg masses, number of eggs (Table 1 and Fig. 2) and average number of eggs per egg mass are highest in the control followed by the $250 \mathrm{ppm}$ and lowest in the $500 \mathrm{ppm}$ treatment. The reproductive period was shorter in the treated snails. 
BIOTROPIA No. 5, 1991/1992

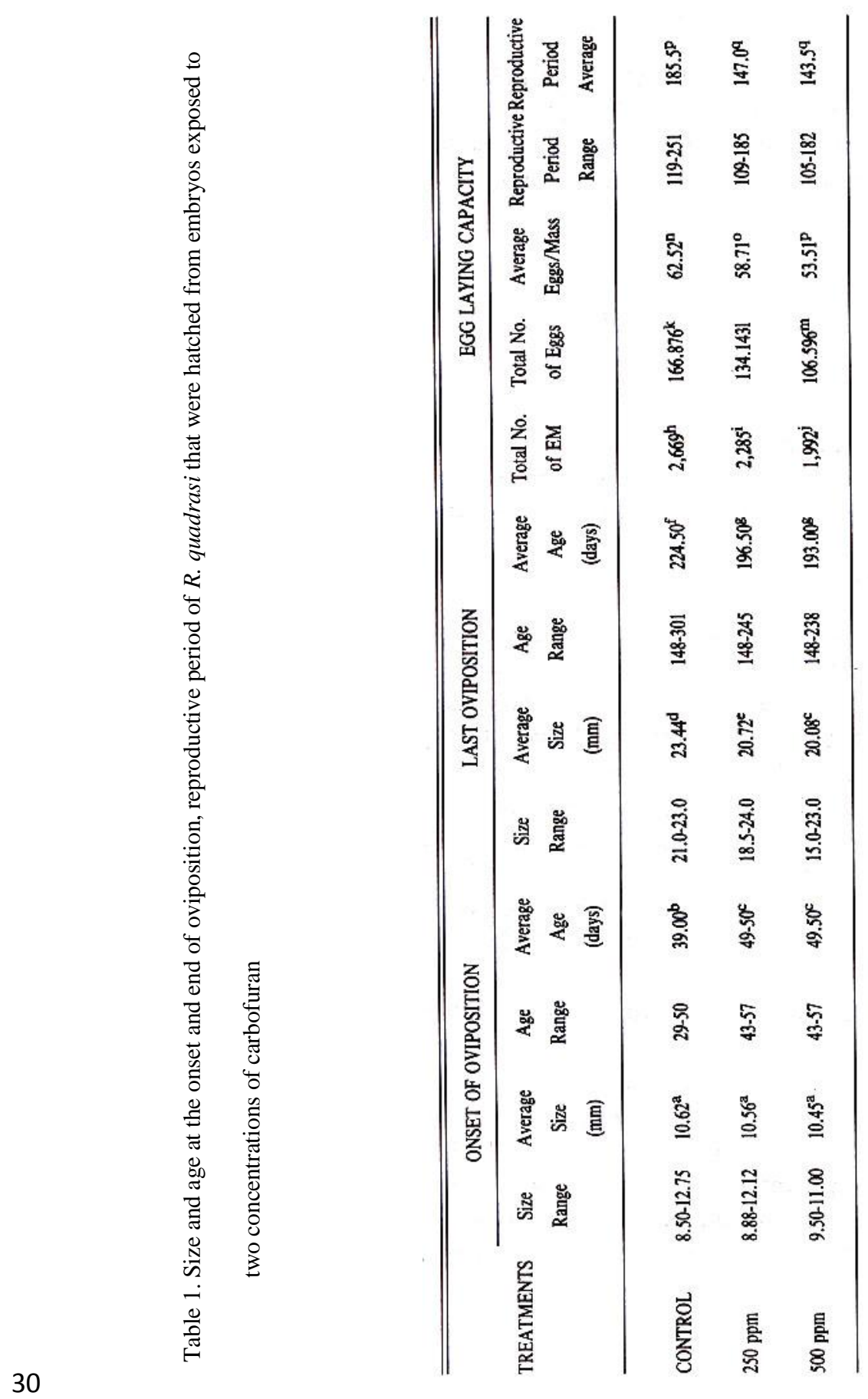


Efects of carbofuran on the reproductive capacity - Imelda F. Pagulayan et al.

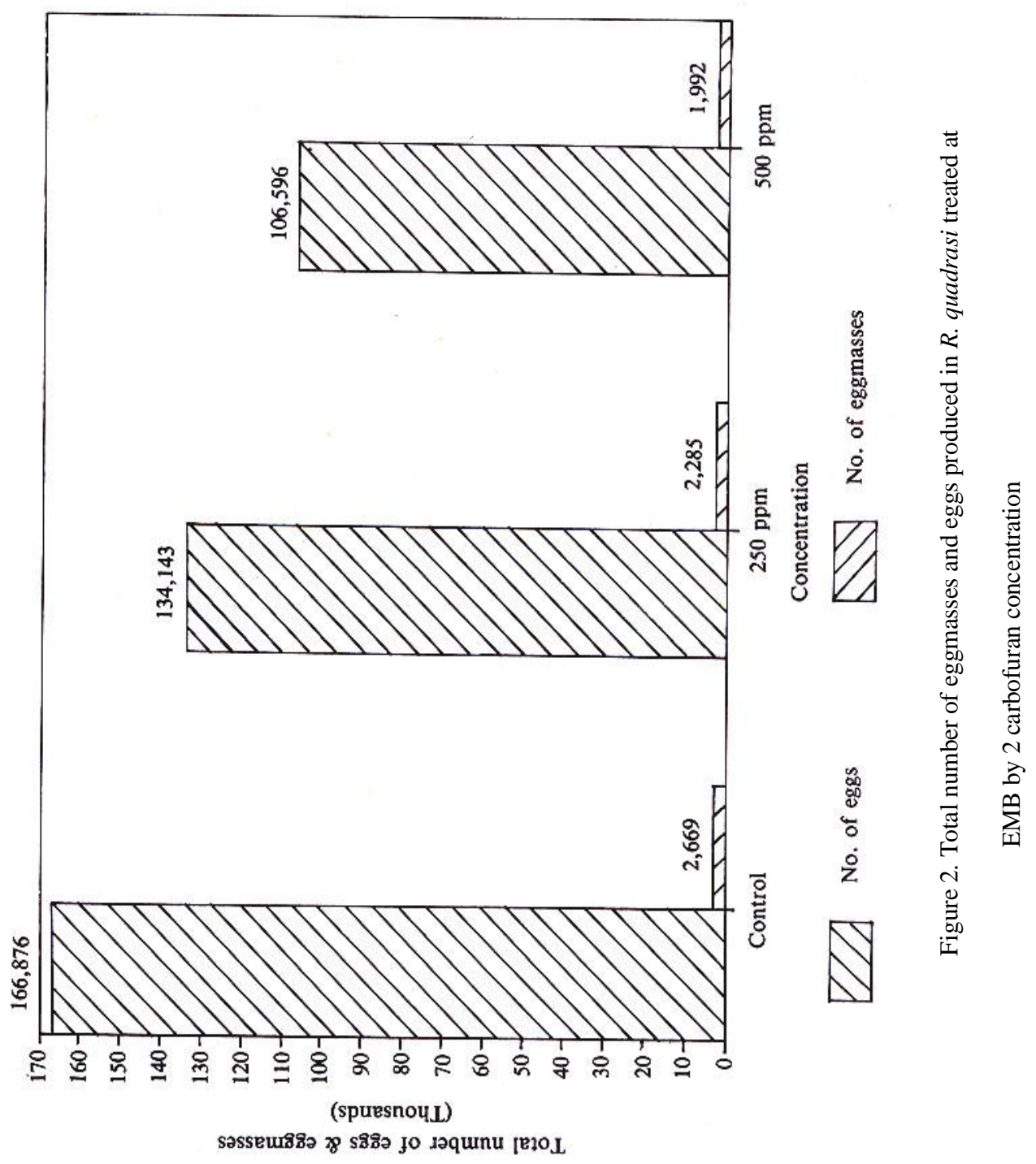




\section{B. Treatment at Week 4, Pre-Sexual Maturity (PSM)}

Table 2 shows the effect on the size and age of the snails treated at the end of week 4 to the end of week 6 at the onset and end of oviposition. The total number of egg masses, eggs (Fig. 3) and the average eggs per egg mass observed during the entire reproductive period is also shown in the table.

With the exception of the 2000 ppm treatment wherein the average snail size at initial oviposition is smaller, the size average of the rest of the treatments and control are not significantly different from each other. The average age for initial oviposition in the control is 40.44 days, while the treated snails at 4 carbofuran concentrations are 53.36, 55.0, 56.5 and 55.84 days. This is an indication that treated snails oviposited at an older age.

The average size in the last oviposition of the treated snails is smaller than in the control except in the 2000 ppm treatment. The average age at last oviposition is higher in the control and significantly lower in the treated. The 500, 1000 and 2000 ppm treatments were not significantly different from each other but significantly different from the 250 ppm group.

The number of egg masses and eggs in the snails exposed to $2000 \mathrm{ppm}$ is significantly low. There is no significant difference though in the total number of eggs and egg masses among the other experimental set-ups including the control (Fig. 3). The reproductive period is longest in the control, and decreasing with higher concentrations. Those exposed to 500, 1000 and 2000 ppm are lower but not significantly different from each other.

\section{Treatment at Week 7, Sexual Maturity (SM)}

Table 3 shows the effect of the 4 carbofuran concentration on the size of the snails treated at week 7 at the onset and end of oviposition. The total number of egg masses laid and the total number of eggs for the entire reproductive period is also shown (Fig. 4). The average number of eggs per egg mass at the end of the observation period is likewise indicated.

It is shown in Table 3 that untreated snails start to oviposit at an average length of $9.6 \mathrm{~mm}$. The average size of the snails at the onset of oviposition in all four treatment groups as well as in the control does not significantly differ from each other. The average age, however, was older in the treated snails, indicating that while sizes are more or less the same, oviposition occurred at an older age. There are snails in the untreated that oviposited in as early as 35 days of age, while the youngest snail that oviposited in the experimental group was 45 days old, or a difference of 10 days. Some snails did not oviposit: 1 snail in the untreated, 10 in the 250 ppm treatment, 8 in the 500 ppm treatment, 6 in the 1000 ppm treatment and 
Effects of carbofuran on the reproductive capacity - Imelda F. Pagulayan et al.

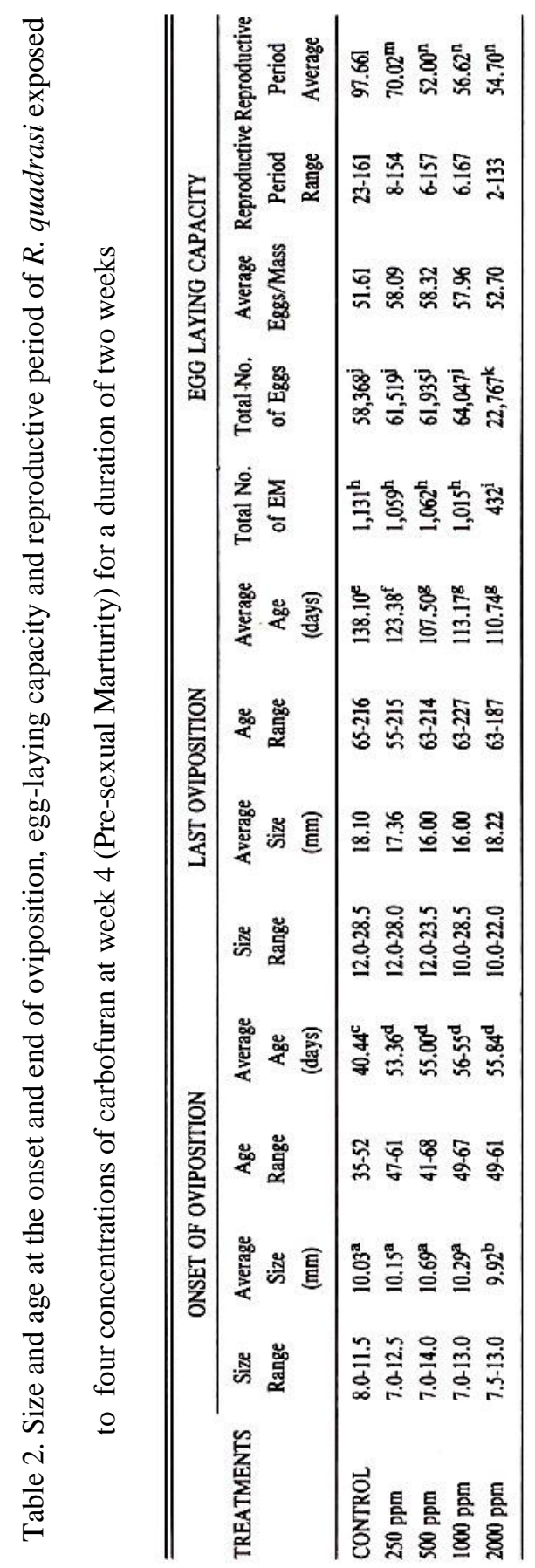

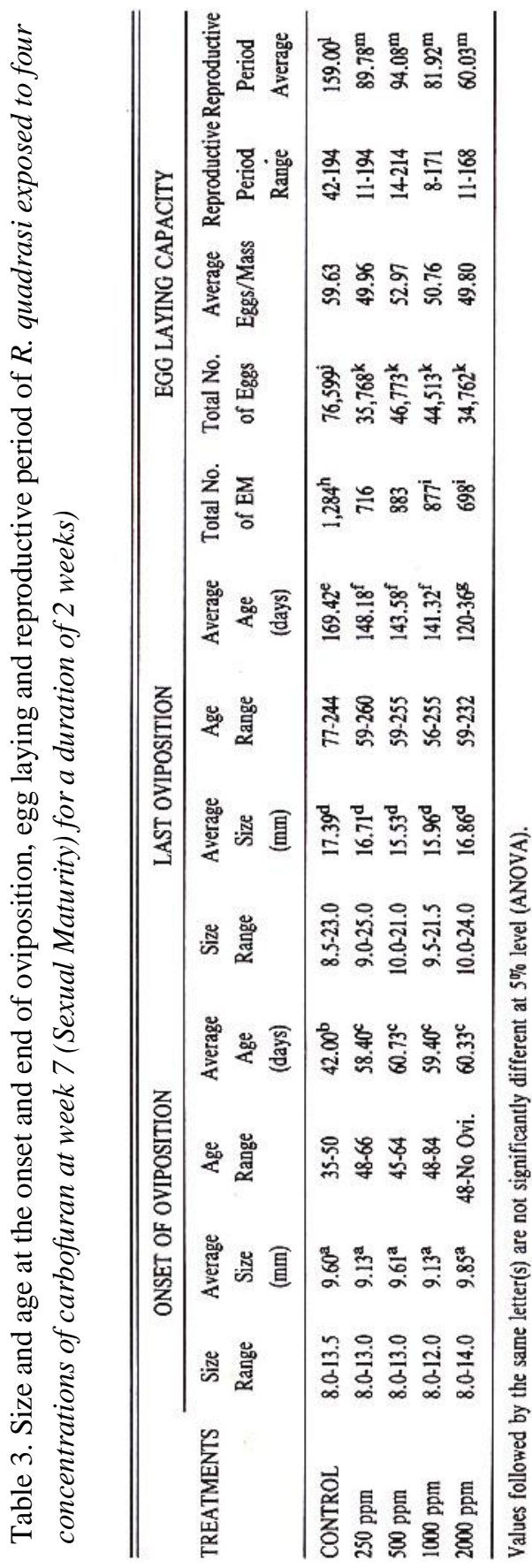


BIOTROPIA No. 5, 1991/1992

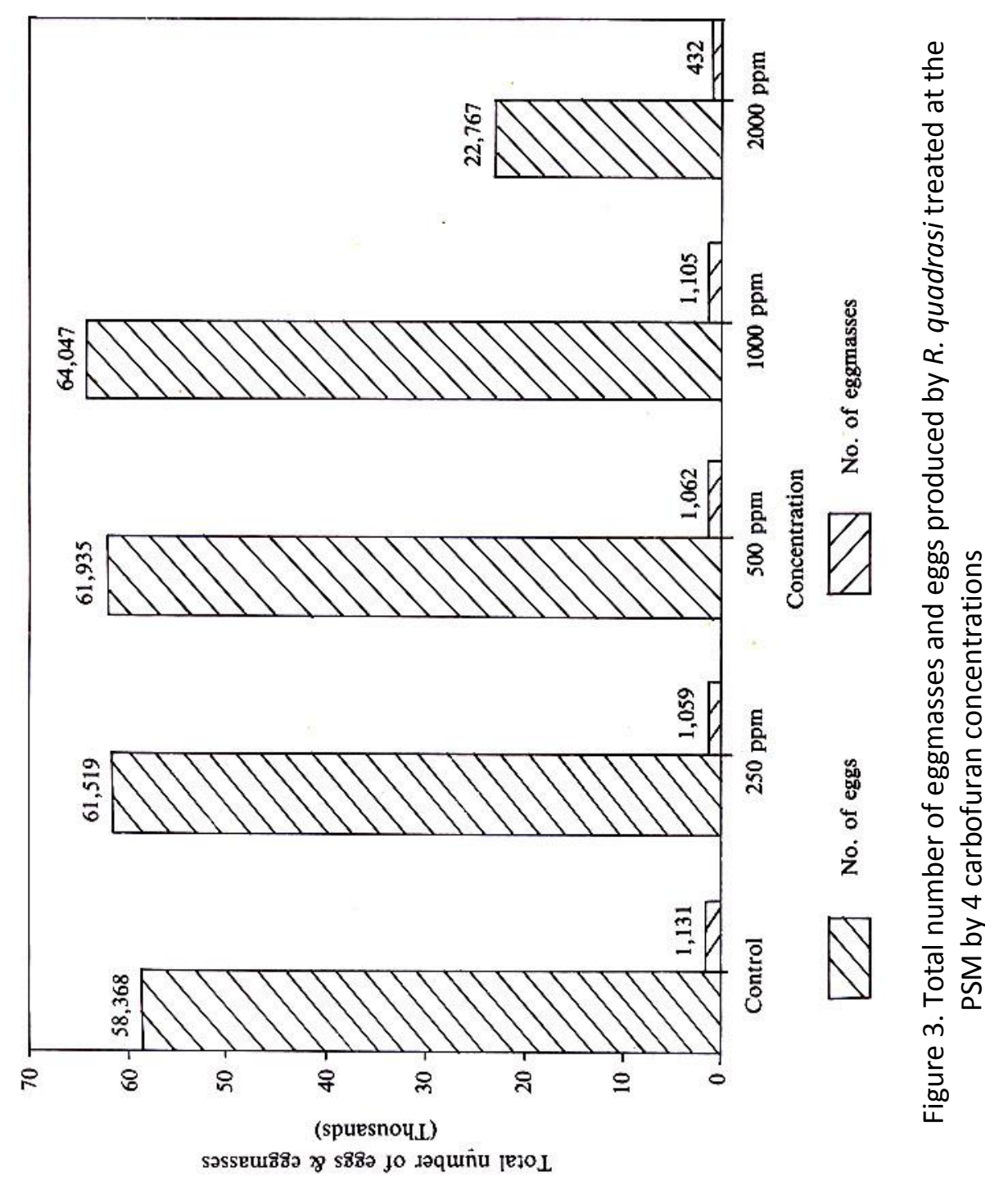


Effects of carbofuran on the reproductive capacity - Imelda F. Pagulayan et al.

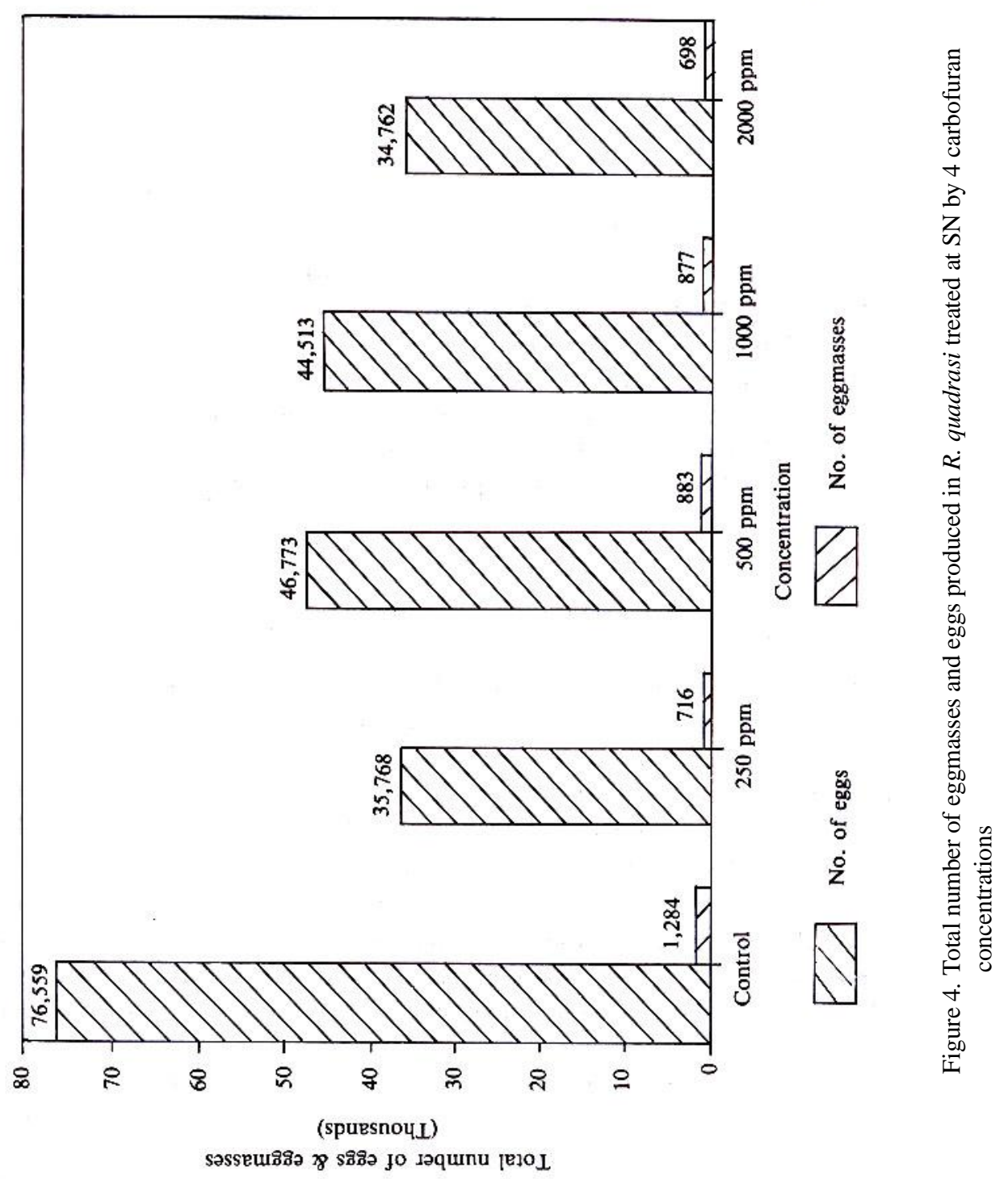


7 in the $2000 \mathrm{ppm}$ treatment. Average age at first oviposition in the untreated is 42 days, while in the treated the range is from 58.4 to 60.73 days (Table 3).

The average size of the snails at last oviposition was smaller in the treated but not significantly different from control and from each other. The average age at last oviposition, however, is highest in the control (169.42 days) and lowest in the $2000 \mathrm{ppm}$ treatment (120.36 days) in a decreasing order with increasing concentration.

The total number of egg masses and eggs was highest in the untreated snails, 1284 and 76559 , respectively. The number of egg masses and eggs produced in the treated was lower and significantly different from the control but not significantly different from each other. The average number of eggs per mass is highest in the control and lower in the treated. The numbers of snails that reached the stage of oviposition in the treated set-ups were reduced.

The reproductive capacity of the treated snails increases weeks after treatment. The observation is more evident in the treated than in the untreated snails. However, the above increase is still not enough to equal the number of egg masses and eggs produced by the control group. The reproductive period in the treated snails is significantly lower than in the control.

\section{Comparative Fecundity Results in the Three Designated Stages (EMB, PSM, SM)}

Table 4 is the summary of the effects of all the treatments on the size and age at the onset and end of oviposition, on the reproductive capacity and length of the reproductive period in the three stages of treatment.

The size range in days at the start of oviposition was 29 - 50 days for the EMB control; 35 - 52 days for the PSM control; and 35 - 50 days in the SM control. The total range using 138 snails was from 29-52 days.

The smallest in size average at the same time oldest in average for the initial oviposition in all the treatments was on the SM treated snails, followed by the PSM and the largest but the youngest to oviposit was in the EMB. While Table 4 shows that the PSM group is the earliest to stop ovipositing, it cannot be concluded that it is the most sensitive to the treatment since the control group follows the same trend. The reproductive capacity is highest in the EMB treated snails. While SM treated snails had a longer reproductive period than the PSM, their reproductive capacity is very much less. 
Effects of carbofuran on the reproduction capacity - Imelda F. Pagulayan et al

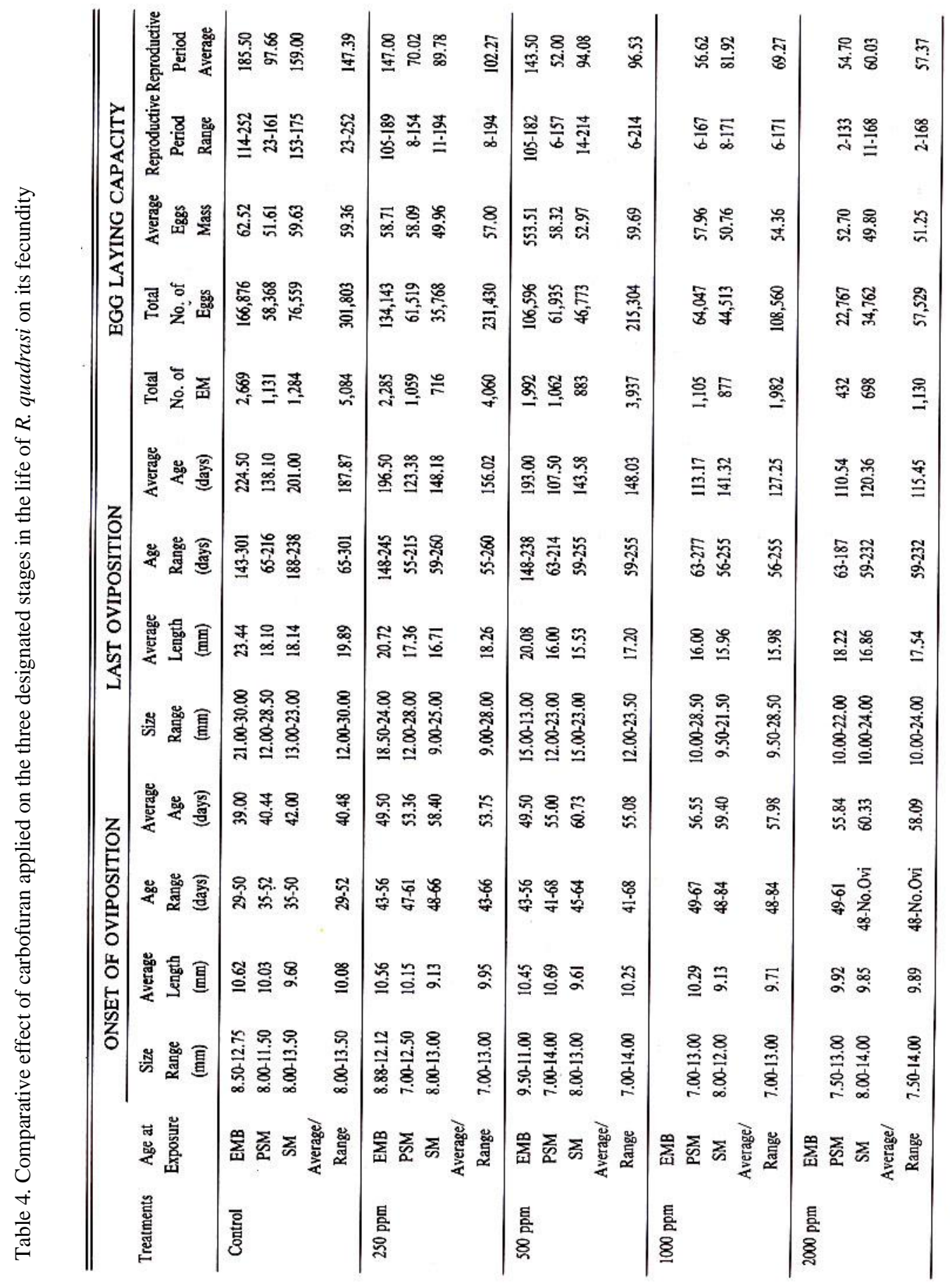




\section{DISCUSSION}

The results of previous work (de Lara and Enriquez 1981) gave a range of 35 -42 days under controlled condition as the start of oviposition. In the current work, however, the total range in these 138 snails was 29-52 days. The current results did not exactly sustain the earlier findings, perhaps because of the effect of cold temperature when these experiments were done, since the expected oviposition periods coincided with the cold months of December and January. Boray (1964) observed in his work that no copulation occurred in L. tomentosa in temperatures below $16^{\circ} \mathrm{C}$ in the laboratory cultures or in the field. The variations apparently reported in the reproductive biology of lymnaeids in general may be due to the different culture methods used. However, Remigio and Pagulayan (1984) contradict this finding.

Start of oviposition was delayed in all the treated snails while size range and average do not differ much in both the untreated and treated snails in the initial oviposition. The treated snails are older which means that it took a greater number days to reach the ovipositing size. It seems that it is more the size rather than age that determines the onset of oviposition. This is true in all the 3 age treatments. Since growth is delayed by the treatment, delay in the oviposition could be attributed to it. It can be stated that the higher the concentration, the later is the start of oviposition. This could also be due to a delay in the rate of gonadal maturation. The higher the concentration, the earlier the cessation of oviposition.

The number of egg masses and eggs produced at initial oviposition is highest at the control and lowest at the 2000 ppm treatment. The treatment not only delayed but also inhibited the reproductive capacity of most of the snails. Inhibition increased with increasing carbofuran concentration. However, the actual number of egg masses- at first oviposition could not be correctly determined. The fact that the number of egg masses at its first appearance is less than the number of snails clearly indicates that not all oviposited. There is no way of determining which of the snails in the basin did or did not oviposit since they are in groups of 4's and 5's. It is possible that the first oviposition in some may have been included in later weeks and counted as later ovipositions. While it is true that there is no accurate method of counting the number of egg masses for first oviposition, there is no other way, unless observations will be done on individual snails cultured in separate basins; such condition, however, is not conducive to fecundity.

The results showed that the carbofuran maybe more effective in a particular age group, and the results could have altered the individual's competitive ability, i.e. food getting, mating. This could be the reason for the decreasing fecundity with increasing concentration in the EMB; there was a drastic effect only 
of the 2000 ppm on the egg laying capacity of the PSM treated snails, and a drastic effect of all the treatments on the egg laying capacity of the SM treated snails. It may also be possible that the PSM treated snails could have recovered after the effect of the 250 , 500 and 1000 ppm carbofuran concentrations had diminished. Recovery brought them back on equal footing with the untreated ones, thus the egg production was higher, counteracting the low egg production at the start. The initial effect, therefore, seems to be delaying in this particular stage instead of totally inhibiting with the exception of the 2000 ppm which partially inhibited the egg laying capacity. Godan (1983) stated that the periods of greatest sensitivity occur when the gastropods are very young and again when they are adults, at the peak of their reproductive activity. Resistance is greatest among juveniles before the onset of sexual maturity. This reinforces the results of this work, as shown by the effect on fecundity of the SM treated snails. Sexual maturity is the most critical stage to carbofuran treatment when it comes to reproductive capacity.

The reproductive period is shorter in the treated snails and is again inversely proportional to the carbofuran concentration. The snails had to attain a bigger size and older age before oviposition took place.

The snails used as control in the EMB, PSM and SM treatments had a wide range of variation for the first oviposition. The said control set-ups were not done at the same time. That could explain the wide variation in 29 - 50 for the EMB control; 35 - 52 for the PSM control; 35 - 50 for the SM control. The final average, though, for all the control is still 40.48 days which still supports the upper range of the results of earlier works, 35-42 days (de Lara and Enriquez 1981).

The pesticide decreased the reproductive capacity of the snails and shortened the reproductive period. Unlike its effect on growth, the SM treated snails were the ones adversely affected. There were snails that did not oviposit at all and a great number did not reach the size/age of oviposition. The carbofuran, depending on the concentration and/or the stage of application must have direct and subtle impacts on behaviour that influences the snail's competitive success i.e. small changes in courtship or mating rituals induced by the carbofuran may lead to mating failure, thus affecting the reproductive capacity and period.

\section{REFERENCES}

ALLMON, F. 1985. Pesticides and unhealthy dependence. Science 6(8): 14.

CREMLYN, R. 1980. Pesticides: preparation and mode of action. John Wiley and Sons, New York.

DE LARA, A.V. and G.L. ENRIQUEZ. 1981. Life history of laboratory reared Radix quadrasi (Gastropoda: Pulmonata).

Kalikasan 10(2-3): 242-254. 
BIOTROPIA No. 5, 1991/1992

GODAN, D. 1983. Pest slugs and snails, biology and control. Springer-Verlag, Berlin.

GUNTHER,F.A., W.E. WESTTAKEand P.S. JAOLAN. 1968. Reported.

HANDA, S.K. 1980. Spectrophotometric determination of carbofuran residues in water. Assoc. Chem. 64(2): 200-201.

MANE, U.H., M.S. KACHOLE, and S.S. PAWAR. 1979. Effect of Pesticides and Narcotants on Bivalve Mollusks. Malac. 18: 347-360.

METCALF, R.L, T.R. FUKOTO, C. COLUNS, K. BERCK, S. ABD EL-AZZ, R. MUNOZ and C.C. CESSRL. 1968. Metabolism of 2,2-dimethyl-2,3-dihydrobenzofuranyl-7-N-methyl carbamate (Furadan) in plants, insects, and mammals. J. Agr. Food. Chem. 16(2): 300-311.

PAGULAYAN, I.F. and D. DARVIN. 1986. The systematic relationship of Radix quadrasi and Mixas cumingiana (Pulmonata: Lymnaeidae). Part III Genetics and Part IV Ecology. Final Report on a Research Project submitted to the Natural Sciences Research Institute, Diliman, Quezon City.

RANGASWANY, J.R., Y.D. VIJAYASHANAKAR, and S.R. PRAKISH. 1975. A simple Spectrophotometric method for the determination of carbofuran residues. J. AOAC 59(6): 1276-1278.

Yu, C., G.M. BOOTH, D.J. HANSEN, and L.R. LARDEN. 1974. Fate of carbofuran in a model ecosystem. J. Food Chem. 22(3): 431-434. 